\title{
Inverse Free Iterative Methods for Nonlinear Ill-Posed Operator Equations
}

\author{
Ioannis K. Argyros, ${ }^{1}$ Santhosh George, ${ }^{2}$ and P. Jidesh ${ }^{2}$ \\ ${ }^{1}$ Department of Mathematical Sciences, Cameron University, Lawton, OK 73505, USA \\ ${ }^{2}$ Department of Mathematical and Computational Sciences, National Institute of Technology Karnataka, Mangalore 575025, India \\ Correspondence should be addressed to Ioannis K. Argyros; iargyros@cameron.edu
}

Received 16 January 2014; Revised 2 April 2014; Accepted 5 April 2014; Published 17 April 2014

Academic Editor: V. R. Khalilov

Copyright (c) 2014 Ioannis K. Argyros et al. This is an open access article distributed under the Creative Commons Attribution License, which permits unrestricted use, distribution, and reproduction in any medium, provided the original work is properly cited.

We present a new iterative method which does not involve inversion of the operators for obtaining an approximate solution for the nonlinear ill-posed operator equation $F(x)=y$. The proposed method is a modified form of Tikhonov gradient (TIGRA) method considered by Ramlau (2003). The regularization parameter is chosen according to the balancing principle considered by Pereverzev and Schock (2005). The error estimate is derived under a general source condition and is of optimal order. Some numerical examples involving integral equations are also given in this paper.

\section{Introduction}

This paper is devoted to the study of nonlinear ill-posed problem

$$
F(x)=y
$$

where $F: D(F) \subseteq X \rightarrow Y$ is a nonlinear operator between the Hilbert spaces $X$ and $Y$. We assume that $F$ is a Fréchetdifferentiable nonlinear operator acting between infinite dimensional Hilbert spaces $X$ and $Y$ with corresponding inner products $\langle\cdot, \cdot\rangle$ and norms $\|\cdot\|$, respectively. Further it is assumed that (1) has a solution $\hat{x}$, for exact data; that is, $F(\widehat{x})=y$, but due to the nonlinearity of $F$ this solution need not be unique. Therefore we consider a $x_{0}$-minimal norm solution of (1). Recall that [1-3] a solution $\widehat{x}$ of (1) is said to be an $x_{0}$-minimal norm $\left(x_{0}\right.$-MNS) solution of (1) if

$$
\begin{gathered}
F(\hat{x})=y, \\
\left\|x_{0}-\widehat{x}\right\|=\min _{x \in D(F)}\left\{\left\|x-x_{0}\right\|: F(x)=y\right\} .
\end{gathered}
$$

In the following, we always assume the existence of an $x_{0}$ MNS for exact data $y$. The element $x_{0} \in X$ in (3) plays the role of a selection criterion [4] and is assumed to be known.
Since (1) is ill-posed, regularization techniques are required to obtain an approximation for $\widehat{x}$. Tikhonov regularization has been investigated by many authors (see e.g., [2, 4, 5]) to solve nonlinear ill-posed problems in a stable manner. In Tikhonov regularization, a solution of the problem (1) is approximated by a solution of the minimization problem

$$
J_{\alpha}(x):=\min _{x \in D(F)}\left\|F(x)-y^{\delta}\right\|^{2}+\alpha\left\|x-x_{0}\right\|^{2},
$$

where $\alpha>0$ is a small regularization parameter and $y^{\delta} \in Y$ is the available noisy data, for which we have the additional information that

$$
\left\|y-y^{\delta}\right\| \leq \delta
$$

It is known [3] that the minimizer $x_{\alpha}^{\delta}$ of the functional $J_{\alpha}(x)$ satisfies the Euler equation

$$
F^{\prime}(x)^{*}\left(F(x)-y^{\delta}\right)+\alpha\left(x-x_{0}\right)=0
$$

of the Tikhonov functional $J_{\alpha}(x)$. Here $F^{\prime}(\cdot)^{*}: Y \rightarrow X$ denotes the adjoint of the Fréchet derivative $F^{\prime}(\cdot): X \rightarrow Y$. It is also known that [1] for properly chosen regularization parameter $\alpha$, the minimizer $x_{\alpha}^{\delta}$ of the functional $J_{\alpha}(x)$ is a 
good approximation to a solution $\widehat{x}$ with minimal distance from $x_{0}$. Thus the main focus is to find a minimizing element $x_{\alpha}^{\delta}$ of the Tikhonov functional (4). But the Tikhonov functional with nonlinear operator $F$ might have several minima, so to ensure the convergence of any optimization algorithm to a global minimizer $x_{\alpha}^{\delta}$ of the Tikhonov functional (3), one has to employ stronger restrictions on the operator [6].

In the last few years many authors considered iterative methods, for example, Landweber method [7, 8], LevenbergMarquardt method [9], Gauss-Newton [10, 11], Conjugate Gradient [12], Newton-like methods [13, 14], and TIGRA (Tikhonov gradient method) [6]. For Landweber's and TIGRA method, one has to evaluate the operator $F$ and the adjoint of the Fréchet derivative of $F$. For all other methods, one has to solve a linear equation additionally.

In [6], Ramlau considered the TIGRA method defined iteratively by

$$
x_{k+1}^{\delta}=x_{k}^{\delta}+\beta_{k}\left[F^{\prime}\left(x_{k}^{\delta}\right)^{*}\left(y^{\delta}-F\left(x_{k}^{\delta}\right)\right)+\alpha_{k}\left(x_{k}^{\delta}-x_{0}\right)\right] \text {, }
$$

where $x_{0}^{\delta}=x_{0}, \beta_{k}$ is a scaling parameter and $\alpha_{k}$ is a regularization parameter, which will change during the iteration and obtained a convergence rate estimate for the TIGRA algorithm under the following assumptions:

(1) $F$ is twice Fréchet differentiable with a continuous second derivative,

(2) the first derivative is Lipschitz continuous:

$$
\left\|F^{\prime}(u)-F^{\prime}(v)\right\| \leq L\|u-v\|,
$$

(3) there exists $w \in Y$ with

$$
x_{0}-\widehat{x}=F^{\prime}(\widehat{x}) w
$$

and

(4) $\|w\| \leq \rho$ and $L \rho \leq 0.241$.

In [15], Scherzer considered a similar iteration procedure under a much stronger condition

$$
\begin{array}{r}
\left\|F(\bar{x})-F(x)-F^{\prime}(x)(x-\bar{x})\right\| \leq \eta\|F(\bar{x})-F(x)\|, \\
\eta<\frac{1}{2} .
\end{array}
$$

In this paper we consider a modified form of iteration (7). Precisely we consider the sequence $\left(x_{n, \alpha}^{\delta}\right)$ defined iteratively by

$$
x_{n+1, \alpha}^{\delta}=x_{n, \alpha}^{\delta}-\left[F^{\prime}\left(x_{0}\right)^{*}\left(F\left(x_{n, \alpha}^{\delta}\right)-y^{\delta}\right)+\alpha\left(x_{n, \alpha}^{\delta}-x_{0}\right)\right],
$$

where $x_{0, \alpha}^{\delta}:=x_{0}, A_{0}=F^{\prime}\left(x_{0}\right)$, and $\alpha$ is the regularization parameter. The regularization parameter $\alpha$ is chosen from a finite set $D=\left\{\alpha_{i}: \alpha_{0}<\alpha_{1}<\cdots<\alpha_{N}<1\right\}$ using the adaptive method considered by Pereverzev and Schock in [16]. We prove that $x_{n, \alpha}^{\delta}$ converges to the unique solution $x_{\alpha}^{\delta}$ (see Theorem 2 ) of the equation

$$
F^{\prime}\left(x_{0}\right)^{*}\left(F(x)-y^{\delta}\right)+\alpha\left(x-x_{0}\right)=0
$$

and that $x_{\alpha}^{\delta}$ is a good approximation for $\hat{x}$. Our approach, in the convergence analysis of the method as well as the choice of the parameters, is different from that of [6].

Note that in TIGRA method (i.e., (7)) the scaling parameter $\beta_{k}$ and the regularization parameter $\alpha_{k}$ will change during the iteration, but in the proposed method no scaling parameter is used and the regularization parameter does not change during the iteration. Also, in the proposed method one needs to compute the Fréchet derivative only at one point $x_{0}$. These are the main advantages of the proposed method.

The organization of this paper is as follows. In Section 2 we provide some preparatory results and Section 3 deals with the convergence analysis of the proposed method. Error bounds under an a priori and under the balancing principle are given in Section 4. Numerical examples involving integral equations are given in Section 5. Finally the paper ends with conclusion in Section 6.

\section{Preparatory Results}

Throughout this paper we assume that the operator $F$ satisfies the following assumptions.

Assumption 1. (a) There exists a constant $k_{0}>0$ such that for every $x \in D(F)$ and $v \in X$, there exists an element $\Phi\left(x, x_{0}, v\right) \in X$ satisfying

$$
\begin{gathered}
{\left[F^{\prime}(x)-F^{\prime}\left(x_{0}\right)\right] v=F^{\prime}\left(x_{0}\right) \Phi\left(x, x_{0}, v\right),} \\
\left\|\Phi\left(x, x_{0}, v\right)\right\| \leq k_{0}\|v\|\left\|x-x_{0}\right\|
\end{gathered}
$$

(b)

$$
\left\|F^{\prime}(x)\right\| \leq m
$$

for all $x \in B_{r}\left(x_{0}\right)$.

Notice that in the literature the stronger than (a) condition

$(\mathrm{a})^{\prime}$

$$
\begin{gathered}
{\left[F^{\prime}(x)-F^{\prime}(z)\right] v=F^{\prime}(z) \xi(x, z, v),} \\
\|\xi(x, z, v)\| \leq K\|v\|\|x-z\|
\end{gathered}
$$

is used for some $\xi(x, z, v) \in X$. However,

$$
k_{0} \leq K
$$

holds in general and $K / k_{0}$ can be arbitrarily large [17]. It is also worth noticing that (a)' implies (a) but not necessarily vice versa and element $\xi$ is less accurate and more difficult to find than $\Phi$ (see also the numerical example).

Next result shows that (12) has a unique solution $x_{\alpha}^{\delta}$ in $B_{r}\left(x_{0}\right)$. 
Theorem 2. Let $\widehat{x} \in B_{r}\left(x_{0}\right) \subset D(F)$ be a solution of (1), Assumption 1 satisfied, and let $F: D(F) \subseteq X \rightarrow Y$ be Fréchet differentiable in a ball $B_{r}\left(x_{0}\right) \subset D(F)$ with radius $r<1 / k_{0}$. Then the regularized problem (12) possesses a unique solution $x_{\alpha}^{\delta}$ in $B_{r}\left(x_{0}\right)$.

Proof. For $x \in B_{r}\left(x_{0}\right)$, let $M_{x}=\int_{0}^{1} F^{\prime}(\widehat{x}+t(x-\widehat{x})) d t$. If $A_{0}^{*} M_{x}+\alpha I$ is invertible, then

$$
\left(A_{0}^{*} M_{x}+\alpha I\right)(x-\widehat{x})=\alpha\left(x_{0}-\widehat{x}\right)+A_{0}^{*}\left(y^{\delta}-y\right)
$$

has a unique solution $x_{\alpha}^{\delta}$ in $B_{r}\left(x_{0}\right)$. Observe that

$$
F(x)-y^{\delta}=F(x)-F(\widehat{x})+y-y^{\delta}=M_{x}(x-\widehat{x})+y-y^{\delta}
$$

and hence

$$
\begin{aligned}
A_{0}^{*}( & \left.F(x)-y^{\delta}\right)+\alpha\left(x-x_{0}\right) \\
& =A_{0}^{*}\left(M_{x}(x-\widehat{x})+y-y^{\delta}\right)+\alpha\left(x-x_{0}\right) \\
& =\left(A_{0}^{*} M_{x}+\alpha I\right)(x-\widehat{x})-\alpha\left(x_{0}-\widehat{x}\right)-A_{0}^{*}\left(y^{\delta}-y\right) .
\end{aligned}
$$

Therefore by (17) $A_{0}^{*}\left(F(x)-y^{\delta}\right)+\alpha\left(x-x_{0}\right)=0$ has a unique solution $x_{\alpha}^{\delta}$ in $B_{r}\left(x_{0}\right)$. So it remains to show that $A_{0}^{*} M_{x}+\alpha I$ is invertible. Note that by Assumption 1, we have

$$
\begin{aligned}
& \left\|\left(A_{0}^{*} A_{0}+\alpha I\right)^{-1} A_{0}^{*}\left(M_{x}-A_{0}\right)\right\| \\
& =\sup _{\|v\| \leq 1}\left\|\left(A_{0}^{*} A_{0}+\alpha I\right)^{-1} A_{0}^{*}\left(M_{x}-A_{0}\right) v\right\| \\
& =\sup _{\|v\| \leq 1}\left\|\left(A_{0}^{*} A_{0}+\alpha I\right)^{-1} A_{0}^{*} \int_{0}^{1}\left(F^{\prime}(\widehat{x}+t(x-\widehat{x}))-A_{0}\right) d t v\right\| \\
& =\sup _{\|v\| \leq 1}\left\|\left(A_{0}^{*} A_{0}+\alpha I\right)^{-1} A_{0}^{*} A_{0} \int_{0}^{1} \Phi\left(\widehat{x}+t(x-\widehat{x}), x_{0}, v\right)\right\| \\
& \leq k_{0} \int_{0}^{1}\left\|\widehat{x}+t(x-\widehat{x})-x_{0}\right\| d t \leq k_{0} r<1 .
\end{aligned}
$$

So $I+\left(A_{0}^{*} A_{0}+\alpha I\right)^{-1} A_{0}^{*}\left(M_{x}-A_{0}\right)$ is invertible. Now from the relation

$$
\begin{aligned}
A_{0}^{*} M_{x} & +\alpha I \\
& =\left(A_{0}^{*} A_{0}+\alpha I\right)\left[I+\left(A_{0}^{*} A_{0}+\alpha I\right)^{-1} A_{0}^{*}\left(M_{x}-A_{0}\right)\right],
\end{aligned}
$$

it follows that $A_{0}^{*} M_{x}+\alpha I$ is invertible.

Assumption 3. There exists a continuous, strictly monotonically increasing function $\varphi:(0, a] \rightarrow(0, \infty)$ with $a \geq$ $\left\|F^{\prime}\left(x_{0}\right)\right\|^{2}$ satisfying

$$
\text { (i) } \lim _{\lambda \rightarrow 0} \varphi(\lambda)=0
$$

(ii)

$$
\sup _{\lambda \geq 0} \frac{\alpha \varphi(\lambda)}{\lambda+\alpha} \leq c_{\varphi} \varphi(\alpha), \quad \forall \lambda \in(0, a] .
$$

(iii) There exists $v \in X$ such that

$$
x_{0}-\widehat{x}=\varphi\left(F^{\prime}\left(x_{0}\right)^{*} F^{\prime}\left(x_{0}\right)\right) v .
$$

One of the crucial results we are going to use to prove our main result is the following theorem.

Theorem 4. Let $0<k_{0} r<1$ and $x_{\alpha}^{\delta}$ be the solution of (12). Then

$$
\left\|x_{\alpha}^{\delta}-\hat{x}\right\| \leq \frac{\max \{1,\|v\|\}}{1-k_{0} r}\left(\frac{\delta}{\sqrt{\alpha}}+\varphi(\alpha)\right) .
$$

Proof. Let $M:=\int_{0}^{1} F^{\prime}\left(\widehat{x}+t\left(x_{\alpha}^{\delta}-\widehat{x}\right)\right) d t$. Then, by fundamental theorem of integration

$$
F\left(x_{\alpha}^{\delta}\right)-F(\widehat{x})=M\left(x_{\alpha}^{\delta}-\widehat{x}\right)
$$

and hence by (12), we have $\left(A_{0}^{*} M+\alpha I\right)\left(x_{\alpha}^{\delta}-\widehat{x}\right)=A_{0}^{*}\left(y^{\delta}-\right.$ $y)+\alpha\left(x_{0}-\widehat{x}\right)$. Thus

$$
\begin{aligned}
x_{\alpha}^{\delta}-\widehat{x}=\left(A_{0}^{*} A_{0}+\alpha I\right)^{-1}[ & A_{0}^{*}\left(y^{\delta}-y\right)+\alpha\left(x_{0}-\widehat{x}\right) \\
& \left.+A_{0}^{*}\left(A_{0}-M\right)\left(x_{\alpha}^{\delta}-\widehat{x}\right)\right] \\
= & s_{1}+s_{2}+s_{3},
\end{aligned}
$$

where $s_{1}:=\left(A_{0}^{*} A_{0}+\alpha I\right)^{-1} A_{0}^{*}\left(y^{\delta}-y\right), s_{2}:=\left(A_{0}^{*} A_{0}+\alpha I\right)^{-1}$ $\alpha\left(x_{0}-\hat{x}\right)$, and $s_{3}:=\left(A_{0}^{*} A_{0}+\alpha I\right)^{-1} A_{0}^{*}\left(A_{0}-M\right)\left(x_{\alpha}^{\delta}-\widehat{x}\right)$. Note that

$$
\left\|s_{1}\right\| \leq \frac{\delta}{\sqrt{\alpha}}
$$

by Assumption 3

$$
\left\|s_{2}\right\| \leq \varphi(\alpha)\|v\|
$$

and by Assumption 1

$$
\begin{aligned}
\left\|s_{3}\right\|= & \left\|\left(A_{0}^{*} A_{0}+\alpha I\right)^{-1} A_{0}^{*}\left(A_{0}-M\right)\left(x_{\alpha}^{\delta}-\widehat{x}\right)\right\| \\
\leq & \|\left(A_{0}^{*} A_{0}+\alpha I\right)^{-1} A_{0}^{*} \\
& \quad \times \int_{0}^{1}\left(A_{0}-F^{\prime}\left(\widehat{x}+t\left(x_{\alpha}^{\delta}-\widehat{x}\right)\right)\right) d t\left(x_{\alpha}^{\delta}-\widehat{x}\right) \| \\
\leq & \|\left(A_{0}^{*} A_{0}+\alpha I\right)^{-1} A_{0}^{*} A_{0} \\
& \times \int_{0}^{1} \Phi\left(\widehat{x}+t\left(x_{\alpha}^{\delta}-\widehat{x}\right), x_{0}, x_{\alpha}^{\delta}-\widehat{x}\right) \| \\
\leq & k_{0} \int_{0}^{1}\left\|\widehat{x}+t\left(x_{\alpha}^{\delta}-\widehat{x}\right)-x_{0}\right\| d t\left\|x_{\alpha}^{\delta}-\widehat{x}\right\| \\
\leq & k_{0} r\left\|x_{\alpha}^{\delta}-\hat{x}\right\| .
\end{aligned}
$$

The result now follows from (26), (27), (28), and (29). 


\section{Convergence Analysis}

In this paper we present a semilocal convergence analysis under the following conditions.

(C1) Suppose $m<\sqrt{1-\alpha_{0}}$. Let

$$
\begin{gathered}
\bar{q}:=1+m^{2} k_{0} r-\alpha_{0}, \\
\bar{\delta}_{0}<\frac{\alpha_{0}^{2}}{4 m^{3} k_{0}} .
\end{gathered}
$$

Let $\left\|x_{0}-\widehat{x}\right\| \leq \rho$ with

$$
\rho \leq \frac{1}{m}\left[\frac{\alpha_{0}^{2}}{4 m^{3} k_{0}}-\bar{\delta}_{0}\right]
$$

and let

$$
\bar{r} \in\left(r_{1}, r_{2}\right),
$$

where $r_{1}=\left(\alpha_{0}-\sqrt{\alpha_{0}^{2}-4 m^{3} k_{0}\left(m \rho+\bar{\delta}_{0}\right)}\right) / 2 m^{2} k_{0}$ and $r_{2}=\min \left\{1 / k_{0}, \alpha_{0} / m^{2} k_{0},\left(\alpha_{0}+\right.\right.$ $\left.\left.\sqrt{\alpha_{0}^{2}-4 m^{3} k_{0}\left(m \rho+\bar{\delta}_{0}\right)}\right) / 2 m^{2} k_{0}\right\}$.

(C2) Suppose $\sqrt{1-\alpha_{0}} \leq m<\sqrt{2-\alpha_{0}}$. Let

$$
\begin{gathered}
q^{*}:=m^{2}\left(1+k_{0} r\right)+\alpha_{0}-1, \\
\delta_{0}^{*}<\frac{\left(2-\alpha_{0}-m^{2}\right)^{2}}{4 m^{3} k_{0}} .
\end{gathered}
$$

Let $\left\|x_{0}-\hat{x}\right\| \leq \rho$ with

$$
\rho \leq \frac{1}{m}\left[\frac{\left(2-\alpha_{0}-m^{2}\right)^{2}}{4 m^{3} k_{0}}-\delta_{0}^{*}\right],
$$

and let

$$
r^{*} \in\left(r_{1}^{*}, r_{2}^{*}\right),
$$

where $r_{1}^{*}=\left(2-\alpha_{0}-m^{2}-\right.$ $\sqrt{\left.\left(2-\alpha_{0}-m^{2}\right)^{2}-4 m^{3} k_{0}\left(m \rho+\delta_{0}^{*}\right)\right)} / 2 m^{2} k_{0} \quad$ and $r_{2}^{*}=\min \left\{1 / k_{0},\left(2-\alpha_{0}-m^{2}\right) / m^{2} k_{0},\left(2-\alpha_{0}-m^{2}+\right.\right.$ $\left.\left.\sqrt{\left(2-\alpha_{0}-m^{2}\right)^{2}-4 m^{3} k_{0}\left(m \rho+\delta_{0}^{*}\right)}\right) / 2 m^{2} k_{0}\right\}$.

Let

$$
\begin{gathered}
\delta_{0}= \begin{cases}\bar{\delta}_{0}, & m<\sqrt{1-\alpha_{0}} \\
\delta_{0}^{*}, & \sqrt{1-\alpha_{0}} \leq m<\sqrt{2-\alpha_{0}},\end{cases} \\
\gamma_{0}=m\left[m \rho+\delta_{0}\right] \\
= \begin{cases}m\left[m \rho+\bar{\delta}_{0}\right], & m<\sqrt{1-\alpha_{0}} \\
m\left[m \rho+\delta_{0}^{*}\right], & \sqrt{1-\alpha_{0}} \leq m<\sqrt{2-\alpha_{0}},\end{cases} \\
q= \begin{cases}\bar{q}, & m<\sqrt{1-\alpha_{0}} \\
q^{*}, & \sqrt{1-\alpha_{0}} \leq m<\sqrt{2-\alpha_{0}},\end{cases}
\end{gathered}
$$

and let

$$
r= \begin{cases}\bar{r}, & m<\sqrt{1-\alpha_{0}} \\ r^{*}, & \sqrt{1-\alpha_{0}} \leq m<\sqrt{2-\alpha_{0}} .\end{cases}
$$

In due course we will make use of the following lemma extensively.

Lemma 5. Let $r$ be as in (38) and $q$ be as in (37). Suppose Assumption 1 holds. Then for $u, v \in B_{r}\left(x_{0}\right)$

$$
\left\|(1-\alpha)(u-v)-A_{0}^{*}(F(u)-F(v))\right\| \leq q\|u-v\| .
$$

Proof. Let $M(u, v):=\int_{0}^{1} F^{\prime}(v+t(u-v)) d t$. Then, by fundamental theorem of integration $F(u)-F(v)=M(u, v)(u-v)$. Hence,

$$
\begin{aligned}
&\left\|(1-\alpha)(u-v)-A_{0}^{*}(F(u)-F(v))\right\| \\
&=\left\|(1-\alpha)(u-v)-A_{0}^{*} M(u, v)(u-v)\right\| \\
&=\left\|\left[(1-\alpha) I-A_{0}^{*}\left(A_{0}+M(u, v)-A_{0}\right)\right](u-v)\right\| \\
& \leq\left\|\left[I-\left(\alpha I+A_{0}^{*} A_{0}\right)\right](u-v)\right\| \\
&+\left\|A_{0}^{*}\left(M(u, v)-A_{0}\right)(u-v)\right\|,
\end{aligned}
$$

so by Assumption 1, we have that

$$
\begin{aligned}
& \left\|(1-\alpha)(u-v)-A_{0}^{*}(F(u)-F(v))\right\| \\
& \quad \leq \sup _{\lambda \in \sigma\left(A_{0}^{*} A_{0}\right)}|1-\alpha-\lambda|\|u-v\| \\
& \quad+\left\|A_{0}^{*} A_{0} \int_{0}^{1} \varphi\left(v+t(u-v), x_{0}, u-v\right)\right\| \\
& \leq\left\{\begin{array}{c}
\left(1-\alpha+m^{2} k_{0} r\right)\|u-v\|, \\
m<\sqrt{1-\alpha_{0}} \\
\left(m^{2}+\alpha-1+m^{2} k_{0} r\right)\|u-v\|, \\
\sqrt{1-\alpha_{0}} \leq m<\sqrt{2-\alpha_{0}}
\end{array}\right. \\
& \leq q\|u-v\| .
\end{aligned}
$$

This completes the proof.

Remark 6. Note that we need $q<1$ for the convergence of the sequence $x_{n, \alpha}^{\delta}$ to $x_{\alpha}^{\delta}$. This in turn forces us to assume that $\bar{q}<1$; that is, $r<\alpha_{0} / m^{2} k_{0}$ (see $r_{2}$ ) and $q^{*}<1$; that is, $r<$ $\left(2-\alpha_{0}-m^{2}\right) / m^{2} k_{0}\left(\right.$ see $\left.r_{2}^{*}\right), m<\sqrt{2-\alpha_{0}}$.

For convenience, we use the notation $x_{n}$ for $x_{n, \alpha}^{\delta}$. Let

$$
e_{n}=\left\|x_{n}-x_{n-1}\right\| .
$$

Remark 7. Observe that

$$
\begin{aligned}
e_{1} & =\left\|A_{0}^{*}\left(F\left(x_{0}\right)-y^{\delta}\right)\right\| \\
& \leq\left\|A_{0}^{*}\left[F\left(x_{0}\right)-F(\widehat{x})+y-y^{\delta}\right]\right\| \\
& \leq\left\|A_{0}^{*}\left[M\left(x_{0}, \widehat{x}\right)\left(x_{0}-\widehat{x}\right)+y-y^{\delta}\right]\right\| \\
& \leq m\left(m\left\|x_{0}-\widehat{x}\right\|+\delta\right) \leq m(m \rho+\delta) \leq \gamma_{0}
\end{aligned}
$$


and $\gamma_{0} /(1-q)=m\left(m \rho+\delta_{0}\right) /(1-q) \leq r$ by the choice of $r$ (this can be seen by substituting $q$ into $m\left(m \rho+\delta_{0}\right) /(1-q)$ and solving the inequality $m\left(m \rho+\delta_{0}\right) /(1-q) \leq r$ for $\left.r\right)$.

Theorem 8. Let $q, r$ be as in (37) and (38), respectively. Then $\left(x_{n}\right)$ defined in (11) is a Cauchy sequence in $B_{r}\left(x_{0}\right)$ and converges to $x_{\alpha}^{\delta} \in \overline{B_{r}\left(x_{0}\right)}$. Further $F^{\prime}\left(x_{0}\right)^{*}\left[F\left(x_{\alpha}^{\delta}\right)-y^{\delta}\right]+\alpha\left(x_{\alpha}^{\delta}-\right.$ $\left.x_{0}\right)=0$ and

$$
\left\|x_{n}-x_{\alpha}^{\delta}\right\| \leq \frac{q^{n}}{1-q} \gamma_{0} .
$$

Proof. Suppose $x_{n} \in B_{r}\left(x_{0}\right)$ for all $n>0$. Then

$$
\begin{aligned}
& x_{n+1}-x_{n} \\
& =x_{n}-x_{n-1}-F^{\prime}\left(x_{0}\right)^{*}\left(F\left(x_{n}\right)-F\left(x_{n-1}\right)\right)-\alpha\left(x_{n}-x_{n-1}\right) \\
& =\left[(1-\alpha)\left(x_{n}-x_{n-1}\right)-A_{0}^{*}\left(F\left(x_{n}\right)-F\left(x_{n-1}\right)\right)\right]
\end{aligned}
$$

and hence by Lemma 5, we have

$$
\left\|x_{n+1}-x_{n}\right\| \leq q\left\|x_{n}-x_{n-1}\right\| \leq q e_{n} .
$$

By Remark 7, $x_{1} \in B_{r}\left(x_{0}\right)$. Now suppose $x_{k} \in B_{r}\left(x_{0}\right)$ for some $k$. Then

$$
\begin{aligned}
\| x_{k+1} & -x_{0} \| \\
& \leq\left\|x_{k+1}-x_{k}\right\|+\left\|x_{k}-x_{k-1}\right\|+\cdots+\left\|x_{1}-x_{0}\right\| \\
& \leq q e_{k}+q e_{k-1}+\cdots+e_{1} \\
& \leq\left(q^{k}+q^{(k-1)}+\cdots+1\right) e_{1} \\
& \leq \frac{1-\left(q^{k+1}\right)}{1-q} e_{1} \\
& \leq \frac{\gamma_{0}}{1-q} \\
& \leq r
\end{aligned}
$$

that is, $x_{k+1} \in B_{r}\left(x_{0}\right)$. Thus by induction $x_{n} \in B_{r}\left(x_{0}\right)$ for all $n>0$. Now we will prove that $\left(x_{n}\right)$ is a Cauchy sequence in $B_{r}\left(x_{0}\right)$. We have

$$
\begin{aligned}
\left\|x_{n+m}-x_{n}\right\| & \leq \sum_{i=0}^{i=m-1}\left\|x_{n+i+1}-x_{n+i}\right\| \\
& \leq \sum_{i=0}^{i=m-1} q e_{n+i} \\
& \leq \sum_{i=0}^{i=m-1} q^{(n+i)} \gamma_{0} \\
& \leq \frac{q^{n}\left(1-q^{m}\right)}{1-q} \gamma_{0} .
\end{aligned}
$$

Thus $x_{n}$ is a Cauchy sequence in $B_{r}\left(x_{0}\right)$ and hence it converges, say to $x_{\alpha}^{\delta} \in \overline{B_{r}\left(x_{0}\right)}$.
By letting $n \rightarrow \infty$ in (11), we obtain $F^{\prime}\left(x_{0}\right)^{*}\left(F\left(x_{\alpha}^{\delta}\right)-y^{\delta}\right)+$ $\alpha\left(x_{\alpha}^{\delta}-x_{0}\right)=0$. Now the result follows by letting $m \rightarrow \infty$ in (48).

Remark 9. (a) Instead of Assumption 1, if we use centerLipschitz conditions; that is,

$$
\left\|F^{\prime}(x)-F^{\prime}\left(x_{0}\right)\right\| \leq L_{0}\left\|x-x_{0}\right\|
$$

holds for all $x \in D(F)$, then one can obtain the estimate

$$
\begin{aligned}
& \left\|(1-\alpha)(u-v)-A_{0}^{*}(F(u)-F(v))\right\| \\
& \quad \leq \begin{cases}1-\alpha+m L_{0} r, & m<\sqrt{1-\alpha_{0}} \\
m^{2}+\alpha-1+m L_{0} r, & \sqrt{1-\alpha_{0}} \leq m<\sqrt{2-\alpha_{0}} .\end{cases}
\end{aligned}
$$

(b) Note that for small enough $k_{0}$ condition (31), (34) is not a severe restriction on $\rho$.

Remark 10. If Assumption 1 is fulfilled only for all $x \in$ $B_{r}\left(x_{0}\right) \cap Q \neq \phi$, where $Q$ is a convex closed a priori set, for which $\widehat{x} \in Q$, then we can modify process (11) by the following way:

$$
x_{n+1, \alpha}^{\delta}=P_{Q}\left(T\left(x_{n, \alpha}^{\delta}\right)\right)
$$

to obtain the same estimate in the following Theorem 11; here $P_{\mathrm{Q}}$ is the metric projection onto the set $Q$ and $T$ is the step operator in (11).

\section{Error Bounds under Source Conditions}

Combining the estimates in Theorems 4 and 8 we obtain the following.

Theorem 11. Let the assumptions in Theorems 4 and 8 hold and let $x_{n, \alpha}^{\delta}$ be as in (11). Then

$$
\left\|x_{n, \alpha}^{\delta}-\hat{x}\right\| \leq \frac{q^{n}}{1-q} \gamma_{0}+\frac{\max \{1,\|v\|\}}{1-k_{0} r}\left(\frac{\delta}{\sqrt{\alpha}}+\varphi(\alpha)\right) .
$$

Further if $n_{\delta}:=\min \left\{n: q^{n}<\delta / \sqrt{\alpha}\right\}$, then

$$
\left\|x_{n_{\delta}, \alpha}^{\delta}-\hat{x}\right\| \leq C\left(\frac{\delta}{\sqrt{\alpha}}+\varphi(\alpha)\right),
$$

where $C:=\gamma_{0} /(1-q)+\max \{1,\|v\|\} /\left(1-k_{0} r\right)$.

4.1. A Priori Choice of the Parameter. Observe that the estimate $\delta / \sqrt{\alpha}+\varphi(\alpha)$ in Theorem 11 is of optimal order for the choice $\alpha:=\alpha_{\delta}$ which satisfies $\delta / \sqrt{\alpha}=\varphi(\alpha)$. Now, using the function $\psi(\lambda):=\lambda \sqrt{\varphi^{-1}(\lambda)}, 0<\lambda \leq a$, we have $\delta=\sqrt{\alpha_{\delta}} \varphi\left(\alpha_{\delta}\right)=\psi\left(\varphi\left(\alpha_{\delta}\right)\right)$ so that $\alpha_{\delta}=\varphi^{-1}\left[\psi^{-1}(\delta)\right]$.

In view of the above observation, Theorem 11 leads to the following.

Theorem 12. Let $\psi(\lambda)=\lambda \sqrt{\varphi^{-1}(\lambda)}, 0<\lambda \leq a$ and assumptions in Theorem 11 hold. For $\delta>0$, let $\alpha_{\delta}=$ $\varphi^{-1}\left[\psi^{-1}(\delta)\right]$ and let $n_{\delta}$ be as in Theorem 11. Then

$$
\left\|x_{n_{\delta}, \alpha_{\delta}}^{\delta}-\hat{x}\right\|=O\left(\psi^{-1}(\delta)\right) .
$$


4.2. Balancing Principle. Note that the a priori choice of the parameter could be achieved only in the ideal situation when the function $\varphi$ is known. The point is that the best function $\varphi$ measuring the rate of convergence in Theorem 11 is usually unknown. Therefore in practical applications different parameters $\alpha=\alpha_{i}$ are often selected from some finite set

$$
D:=\left\{\alpha_{i}: 0<\alpha_{0}<\alpha_{1}<\cdots<\alpha_{N}<1\right\}
$$

and corresponding elements $x_{n, \alpha_{i}}^{\delta}, i=1,2, \ldots, N$ are studied on line. Let

$$
n_{i}:=\min \left\{n: q^{n} \leq \frac{\delta}{\sqrt{\alpha_{i}}}\right\}
$$

and let $x_{\alpha_{i}}^{\delta}:=x_{n_{i}, \alpha_{i}}^{\delta}$. Then from Theorem 11, we have

$$
\left\|x_{\alpha_{i}}^{\delta}-\hat{x}\right\| \leq C\left(\frac{\delta}{\sqrt{\alpha_{i}}}+\varphi\left(\alpha_{i}\right)\right), \quad \forall i=1,2, \ldots, N
$$

We consider the balancing principle suggested by Pereverzev and Schock [16], for choosing the regularization parameter $\alpha$ from the set $D_{N}$ defined by

$$
D_{N}:=\left\{\alpha_{i}=\mu^{i} \alpha_{0}<1, i=1,2, \ldots, N\right\},
$$

where $\alpha_{0}=c \sqrt{\delta}$ for some constant $c$ (see [18]) and $\mu>1$.

To obtain a conclusion from this parameter choice we considered all possible functions $\varphi$ satisfying Assumption 1 and $\varphi\left(\alpha_{i}\right) \leq \delta / \sqrt{\alpha_{i}}$. Any of such functions is called admissible for $\widehat{x}$ and it can be used as a measure for the convergence of $x_{\alpha_{i}}^{\delta} \rightarrow \widehat{x}$ (see [19]).

The main result of this section is the following theorem, proof of which is analogous to the proof of Theorem 4.4 in [13].

Theorem 13. Assume that there exists $i \in\{0,1, \ldots, N\}$ such that $\varphi(\delta) \leq \delta / \sqrt{\alpha_{i}}$. Let assumptions of Theorem 11 be satisfied and let

$$
\begin{gathered}
l:=\max \left\{i: \varphi\left(\alpha_{i}\right) \leq \frac{\delta}{\sqrt{\alpha_{i}}}\right\}<N, \\
k=\max \left\{i: \forall j=1,2, \ldots, i ;\left\|x_{\alpha_{i}}^{\delta}-x_{\alpha_{j}}^{\delta}\right\| \leq 4 C \frac{\delta}{\sqrt{\alpha_{j}}}\right\},
\end{gathered}
$$

where $C$ is as in Theorem 11. Then $l \leq k$ and

$$
\left\|x_{\alpha_{k}}^{\delta}-\widehat{x}\right\| \leq 6 C \mu \psi^{-1}(\delta) .
$$

\section{Numerical Examples}

Let the half-space be modeled by two layers of constant different densities $\sigma_{1}, \sigma_{2}$, separated by a surface $S$ to be determined. In the Cartesian coordinate system, whose plane $x O y$ coincides with the ground surface and the axis $z$ is directed downward, the inverse gravimetry problem has the form (see [20] and References in it)

$$
\begin{aligned}
& \Gamma \Delta \sigma \iint_{D} \frac{1}{\left[\left(x-x^{\prime}\right)^{2}+\left(y-y^{\prime}\right)^{2}+H^{2}\right]^{1 / 2}} d x^{\prime} d y^{\prime} \\
& \quad-\iint_{D} \frac{1}{\left[\left(x-x^{\prime}\right)^{2}+\left(y-y^{\prime}\right)^{2}+u^{2}\left(x^{\prime}, y^{\prime}\right)\right]^{1 / 2}} d x^{\prime} d y^{\prime} \\
& \quad=\Delta g(x, y)
\end{aligned}
$$

here $\Gamma$ is gravity constant, $\Delta=\sigma_{2}-\sigma_{1}$ is the density jump at the interface $S$, described by the function $u(x, y)$ to be evaluated, and $\Delta g(x, y)$ is the anomalous gravitational field caused by deviation of the interface $S$ from horizontal asymptotic plane $z=H$; that is, for the actual solution $\widehat{u}(x, y)$ the following relation holds:

$$
\lim _{|x|,|y| \rightarrow \infty}|\widehat{u}(x, y)-H|=0
$$

$\Delta g(x, y)$ is given on the domain $D$.

Since in (61) the first term does not depend on $u(x, y)(61)$ can be written as

$$
\begin{aligned}
F(u) & \equiv-\iint_{D} \frac{1}{\left[\left(x-x^{\prime}\right)^{2}+\left(y-y^{\prime}\right)^{2}+u^{2}\left(x^{\prime}, y^{\prime}\right)\right]^{1 / 2}} d x^{\prime} d y^{\prime} \\
& =f(x, y)
\end{aligned}
$$

where $f(x, y)=\Delta g(x, y)+F(H)$.

The derivative of the operator $F$ at the point $u_{0}(x, y)$ is expressed by the formula

$$
\begin{aligned}
& F^{\prime}\left(u_{0}\right) h \\
& \quad=\iint_{D} \frac{u_{0}\left(x^{\prime}, y^{\prime}\right) h\left(x^{\prime}, y^{\prime}\right)}{\left[\left(x-x^{\prime}\right)^{2}+\left(y-y^{\prime}\right)^{2}+\left(u_{0}\left(x^{\prime}, y^{\prime}\right)\right)^{2}\right]^{3 / 2}} d x^{\prime} d y^{\prime} .
\end{aligned}
$$

Applying to the integral equations (63) two-dimensional analogy of rectangle's formula with uniform grid for every variable, we obtain the following system of nonlinear equations:

$$
\begin{aligned}
\sum_{i=1}^{m_{2}} \sum_{j=1}^{m_{1}} & \frac{1}{\left[\left(x_{k}-x_{j}^{\prime}\right)^{2}+\left(y_{l}-y_{i}^{\prime}\right)^{2}+u^{2}\left(x_{j}^{\prime}, y_{i}^{\prime}\right)\right]^{1 / 2}} \Delta x \Delta y \\
& =f\left(x_{k}, y_{l}\right) ;
\end{aligned}
$$

$\left(k=1,2, \ldots, m_{1}, l=1,2, \ldots, m_{2}\right)$ for the unknown vector $\left\{u_{j, i}=u\left(x_{j}, y_{i}\right), j=1,2, \ldots, m_{1}, i=1,2, \ldots, m_{2}\right\}$ in vectormatrix form this system takes the form

$$
F_{n}\left(u_{n}\right)=f_{n}
$$

where $u_{n}, f_{n}$ are vectors of dimension $n=m_{1} m_{2}$. 
The discrete variant of the derivative $F^{\prime}\left(u_{0}\right)$ has the form

$$
\begin{aligned}
& \left\{F_{n}^{0} h_{n}\right\}_{k, l} \\
& \quad=\sum_{i=1}^{m_{2}} \sum_{j=1}^{m_{1}} \frac{\Delta x \Delta y u_{0}\left(x_{j}^{\prime}, y_{i}^{\prime}\right) h\left(x_{j}^{\prime}, y_{i}^{\prime}\right)}{\left[\left(x_{k}-x_{j}^{\prime}\right)^{2}+\left(y_{l}-y_{i}^{\prime}\right)^{2}+u_{0}^{2}\left(x_{j}^{\prime}, y_{i}^{\prime}\right)\right]^{3 / 2}},
\end{aligned}
$$

where $u_{0}(x, y)=H$ is constant and $F_{n}^{0}$ is symmetric matrix, for which the component with member $(k, l)$ is evaluated by formula (67).

Let us define the exact solution as

$$
\begin{aligned}
\widehat{u}(x, y)= & 5-2 \exp -\left[\left(\frac{x}{10}-3.5\right)^{2}\left(\frac{y}{10}-2.5\right)^{2}\right] \\
& -3 \exp -\left[\left(\frac{x}{10}-5.5\right)^{2}\left(\frac{y}{10}-4.5\right)^{2}\right],
\end{aligned}
$$

where $\widehat{u}(x, y)$ is given on the domain $D=\left\{0 \leq x \leq m_{1}, 0 \leq\right.$ $\left.y \leq m_{2}\right\}$. Let $\Delta x=\Delta y=1, n=m_{1} \cdot m_{2}, \Delta=0.25, H=5$.

Note that on the set

$$
Q=\{1.0 \leq u(x, y) \leq 10.0\}
$$

$\left\|F^{\prime}(x)-F^{\prime}\left(x_{0}\right)\right\| \leq L_{0}\left\|x-x_{0}\right\|$ (see Remarks 9 and 10 ) satisfied (see $[20,21])$. Besides, for our data $F_{n}^{0}$ is symmetric and positive definite matrix with the minimal eigenvalue $\lambda_{\min } \simeq$ 0.0007 and the condition number $\operatorname{Cond}\left(F_{n}^{0}\right) \simeq 12206$.

It means that for the matrix $F_{n}^{0}$ approximating the operator $F^{\prime}\left(u_{0}\right)$, we have taken initial guess $u_{0}=5$.

Observe that

$$
\begin{aligned}
& u_{0}-\widehat{u} \\
& =2 \exp -\left[\left(\frac{x}{10}-3.5\right)^{2}\left(\frac{y}{10}-2.5\right)^{2}\right] \\
& \quad+3 \exp -\left[\left(\frac{x}{10}-5.5\right)^{2}\left(\frac{y}{10}-4.5\right)^{2}\right] \\
& =\frac{1}{|D|} \iint_{D}\left[2 \exp -\left[\left(\frac{x}{10}-3.5\right)^{2}\left(\frac{y}{10}-2.5\right)^{2}\right]\right. \\
& =\frac{1}{|D|} \iint_{D} \frac{u_{0}\left(x^{\prime}, y^{\prime}\right) h\left(x^{\prime}, y^{\prime}\right)}{\left[\left(x-x^{\prime}\right)^{2}+\left(y-y^{\prime}\right)^{2}+\left(u_{0}\left(x^{\prime}, y^{\prime}\right)\right)^{2}\right]^{3 / 2}} d x^{\prime} d y^{\prime} \\
& =F^{\prime}\left(u_{0}\right) h, \quad
\end{aligned}
$$

where

$$
\begin{aligned}
h\left(x^{\prime}, y^{\prime}\right)= & \frac{\left[\left(x-x^{\prime}\right)^{2}+\left(y-y^{\prime}\right)^{2}+\left(u_{0}\left(x^{\prime}, y^{\prime}\right)\right)^{2}\right]^{3 / 2}}{u_{0}\left(x^{\prime}, y^{\prime}\right)} \\
& \times\left[2 \exp -\left[\left(\frac{x}{10}-3.5\right)^{2}\left(\frac{y}{10}-2.5\right)^{2}\right]\right. \\
& \left.+3 \exp -\left[\left(\frac{x}{10}-5.5\right)^{2}\left(\frac{y}{10}-4.5\right)^{2}\right]\right] .
\end{aligned}
$$

Now since $F^{\prime}\left(u_{0}\right)$ is self-adjoint, we have

$$
u_{0}-\widehat{u}=\left(F^{\prime}\left(u_{0}\right)^{*} F^{\prime}\left(u_{0}\right)\right)^{1 / 2} h
$$

and hence Assumption 3 satisfies for $\varphi(\lambda)=\lambda^{1 / 2}$.

The results of numerical experiments are presented in Table 1 . Here $\widetilde{u}_{n}$ is the numerical solution obtained by our method; the relative error of solution and residual

$$
\Delta_{1}=\frac{\left\|\widehat{u}-\widetilde{u}_{n}\right\|}{\left\|\widehat{u}_{n}\right\|}, \quad \Delta_{2}=\frac{\left\|F_{n}\left(\widetilde{u}_{n}\right)-f_{n}\right\|}{\left\|f_{n}\right\|}
$$

for a noisy right-hand side.

Next we present an example for nonlinear equations where Assumption (a) is satisfied but not (a)'.

Example 1. Let $X=Y=\mathbb{R}, D=[0, \infty), x_{0}=1$ and define function $F$ on $D$ by

$$
F(x)=\frac{x^{1+1 / i}}{1+1 / i}+c_{1} x+c_{2}
$$

where $c_{1}, c_{2}$ are real parameters and $i>2$ an integer. Then $F^{\prime}(x)=x^{1 / i}+c_{1}$ is not Lipschitz on $D$. Hence, Assumption $(a)^{\prime}$ is not satisfied. However central Lipschitz condition Assumption (a) holds.

Indeed, we have

$$
\begin{aligned}
{\left[F^{\prime}(x)\right.} & \left.-F^{\prime}\left(x_{0}\right)\right] 1 \\
& =x^{1 / i}-x_{0}^{1 / i} \\
& =\left(x_{0}^{1 / i}+c_{1}\right) \frac{x-x_{0}}{\left(x_{0}^{1 / i}+c_{1}\right)\left(x_{0}^{(i-1) / i}+\cdots+x^{(i-1) / i}\right)} \\
& =F^{\prime}\left(x_{0}\right) \phi\left(x, x_{0}, 1\right),
\end{aligned}
$$

where $\phi\left(x, x_{0}, 1\right)=\left(x-x_{0}\right) /\left(x_{0}^{1 / i}+c_{1}\right)\left(x_{0}^{(i-1) / i}+\cdots+x^{(i-1) / i}\right)$ and

$$
\left\|\phi\left(x, x_{0}, 1\right)\right\| \leq k_{0}\left|x-x_{0}\right|,
$$

where $k_{0} \geq\left|1 /\left(x_{0}^{1 / i}+c_{1}\right)\left(x_{0}^{(i-1) / i}+\cdots+x^{(i-1) / i}\right)\right|$.

\section{Conclusion}

We have considered an iterative method which does not involve inversion of operator, for obtaining approximate 
TABLE 1: Mesh size and corresponding errors.

\begin{tabular}{lccccc}
\hline$\delta$ & $\alpha_{k}$ & $m_{1}$ & $m_{2}$ & $\Delta_{1}$ & $\Delta_{2}$ \\
\hline \multirow{3}{*}{0.01} & $5.0625 e-04$ & 10 & 11 & 0.0022 & 0.0014 \\
& & 16 & 0.0030 & 0.0022 \\
& & 19 & 20 & 0.0025 & 0.0024 \\
\hline \multirow{3}{*}{0.0044} & $1.0000 e-04$ & 15 & 11 & 0.0012 & $7.1247 e-04$ \\
& & 19 & 20 & 0.0025 & 0.0017 \\
& & 10 & 11 & $6.9788 e-04$ & $4.1727 e-04$ \\
0.0025 & $3.1641 e-05$ & 15 & 16 & 0.0022 & 0.0014 \\
& & 19 & 20 & 0.0025 & 0.0024 \\
\hline
\end{tabular}

solution for a nonlinear ill-posed operator equation $F(x)=y$ when the available data is $y^{\delta}$ in place of the exact data $y$. It is assumed that $F$ is Fréchet differentiable. The procedure involves finding the fixed point of the function

$$
G(x)=x-\left[F^{\prime}\left(x_{0}\right)^{*}\left(F(x)-y^{\delta}\right)+\alpha\left(x-x_{0}\right)\right]
$$

in an iterative manner. For choosing the regularization parameter $\alpha$ we made use of the adaptive method suggested in [16].

\section{Conflict of Interests}

The authors declare that there is no conflict of interests regarding the publication of this paper.

\section{References}

[1] H. W. Engl, M. Hanke, and A. Neubauer, Regularization of Inverse Problems, Kluwer, Dordrecht, The Netherlands, 1996.

[2] Q.-N. Jin and Z.-Y. Hou, "On an a posteriori parameter choice strategy for tikhonov regularization of nonlinear ill-posed problems," Numerische Mathematik, vol. 83, no. 1, pp. 139-159, 1999.

[3] U. Tautenhahn and Q.-N. Jin, "Tikhonov regularization and a posteriori rules for solving nonlinear ill posed problems," Inverse Problems, vol. 19, no. 1, pp. 1-21, 2003.

[4] H. W. Engl, K. Kunisch, and A. Neubauer, "Convergence rates for Tikhonov regularisation of non-linear ill-posed problems," Inverse Problems, vol. 5, no. 4, article 007, pp. 523-540, 1989.

[5] A. Neubauer, "Tikhonov regularisation for non-linear ill-posed problems: optimal convergence rates and finite-dimensional approximation," Inverse Problems, vol. 5, no. 4, article 008, pp. 541-557, 1989.

[6] R. Ramlau, "TIGRA-an iterative algorithm for regularizing nonlinear ill-posed problems," Inverse Problems, vol. 19, no. 2, pp. 433-465, 2003.

[7] M. Hanke, A. Neubauer, and O. Scherzer, "A convergence analysis of the Landweber iteration for nonlinear ill-posed problems," Numerische Mathematik, vol. 72, pp. 21-37, 1995.

[8] R. Ramlau, "Modified Landweber method for inverse problems," Numerical Functional Analysis and Optimization, vol. 20, no. 1, pp. 79-98, 1999.
[9] M. Hanke, "A regularizing Levenberg-Marquardt scheme, with applications to inverse groundwater filtration problems," Inverse Problems, vol. 13, no. 1, pp. 79-95, 1997.

[10] A. B. Bakushinskii, "The problem of the convergence of the iteratively regularized Gauss-Newton method," Computational Mathematics and Mathematical Physics, vol. 32, no. 9, pp. 13531359, 1992.

[11] B. Blaschke, A. Neubauer, and O. Scherzer, "On convergence rates for the iteratively regularized Gauss-Newton method," IMA Journal of Numerical Analysis, vol. 17, no. 3, pp. 421-436, 1997.

[12] M. Hanke, "Regularizing properties of a truncated Newtoncg algorithm for nonlinear inverse problems," Numerical Functional Analysis and Optimization, vol. 18, no. 9-10, pp. 971-993, 1997.

[13] S. George, "On convergence of regularized modified Newton's method for nonlinear ill-posed problems," Journal of Inverse and Ill-Posed Problems, vol. 18, no. 2, pp. 133-146, 2010.

[14] B. Kaltenbacher, "Some Newton-type methods for the regularization of nonlinear ill-posed problems," Inverse Problems, vol. 13, no. 3, pp. 729-753, 1997.

[15] O. Scherzer, "A convergence analysis of a method of steepest descent and a two-step algorithm for nonlinear ill-posed problems," Numerical Functional Analysis and Optimization, vol. 17, no. 1-2, pp. 197-214, 1996.

[16] S. Pereverzev and E. Schock, "On the adaptive selection of the parameter in regularization of ill-posed problems," SIAM Journal on Numerical Analysis, vol. 43, no. 5, pp. 2060-2076, 2005.

[17] I. K. Argyros, Convergence and Applications of Newton-Type Iterations, Springer, New York, NY, USA, 2008.

[18] E. V. Semenova, "Lavrentiev regularization and balancing principle for solving ill-posed problems with monotone operators," Computational Methods in Applied Mathematics, vol. 10, no. 4, pp. 444-454, 2010.

[19] S. Lu and S. V. Pereverzev, "Sparsity reconstruction by the standard Tikhonov method," RICAM-Report, 2008.

[20] V. V. Vasin, I. I. Prutkin, M. Timerkhanova, and L. Yu, "Retrieval of a three-dimensional relief of geological boundary from gravity data," Izvestiya, Physics of the Solid Earth, vol. 32, no. 11, pp. 58-62, 1996.

[21] V. V. Vasin, "Modified processes of Newton type generating Fejer approximations of regularized solutions of nonlinear equations," Proceedings in Mathematics and Machanics, vol. 19, no. 2, pp. 85-97, 2013 (Russian). 


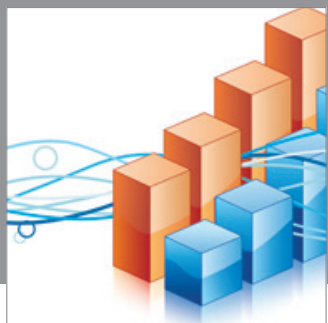

Advances in

Operations Research

mansans

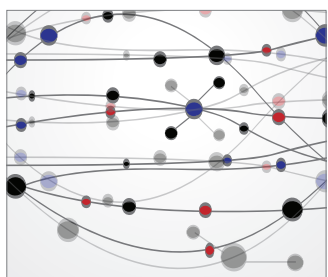

The Scientific World Journal
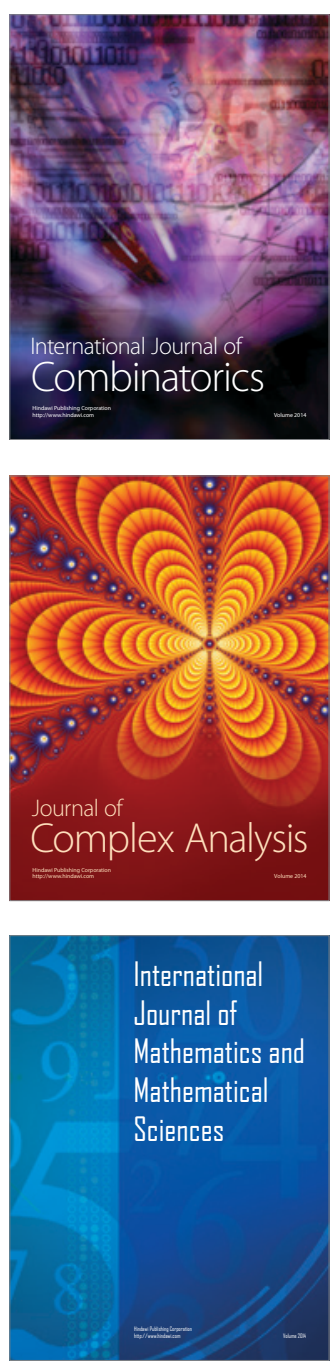
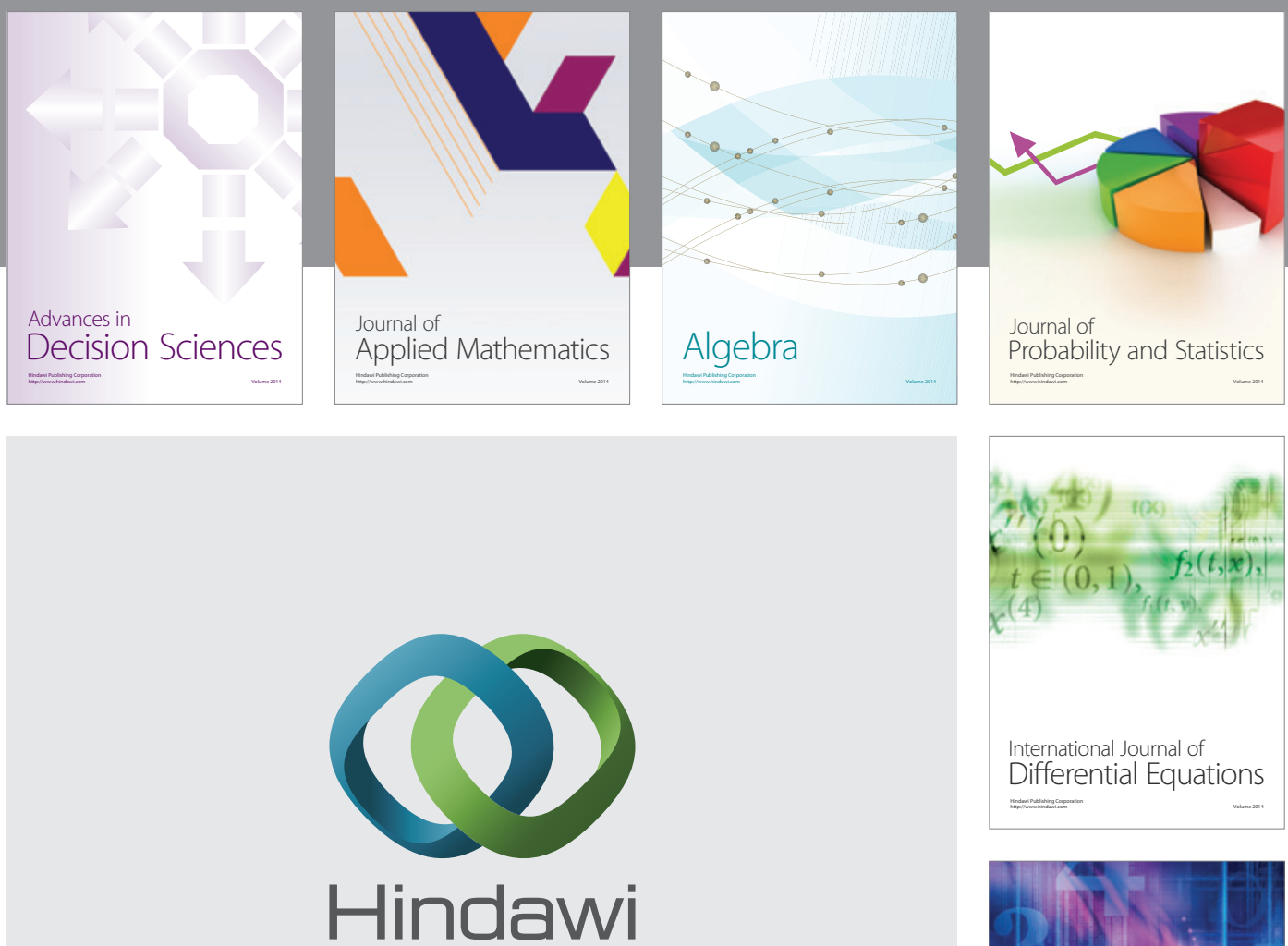

Submit your manuscripts at http://www.hindawi.com
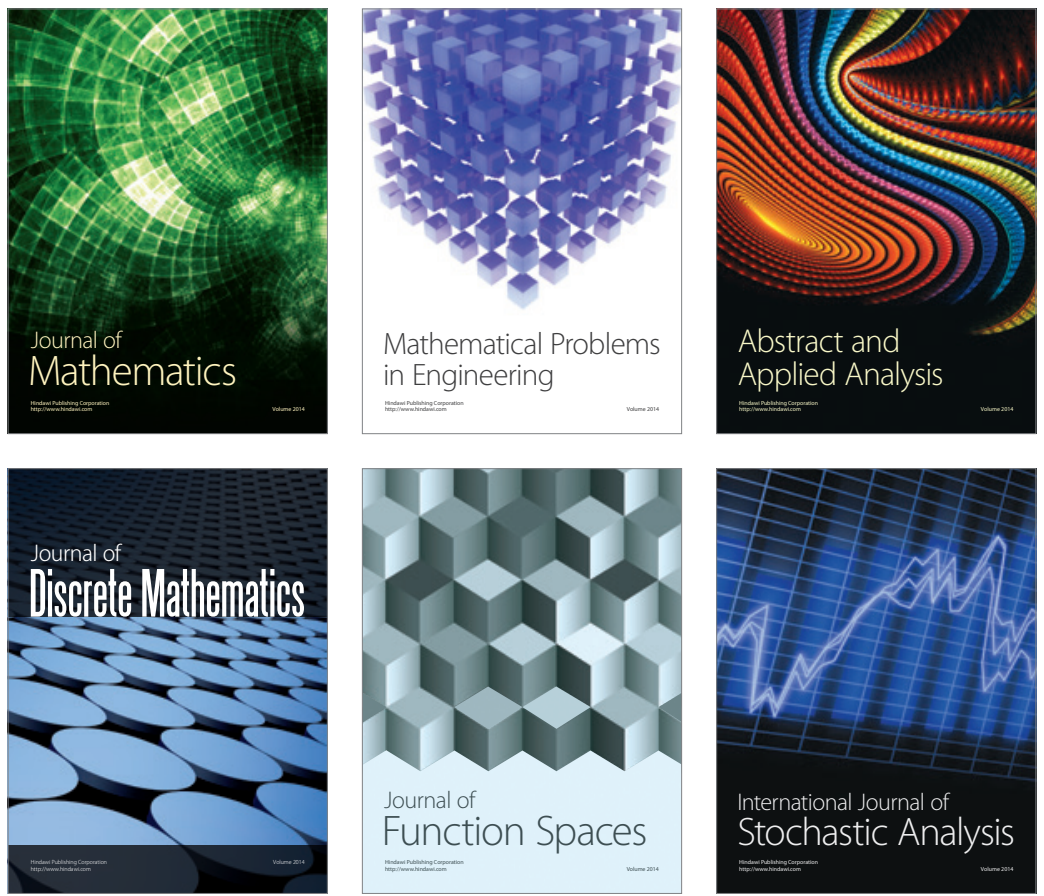

Journal of

Function Spaces

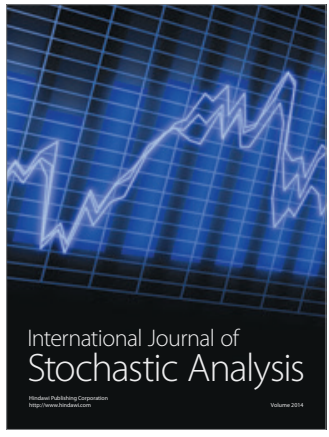

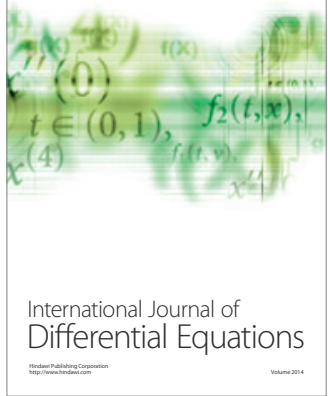
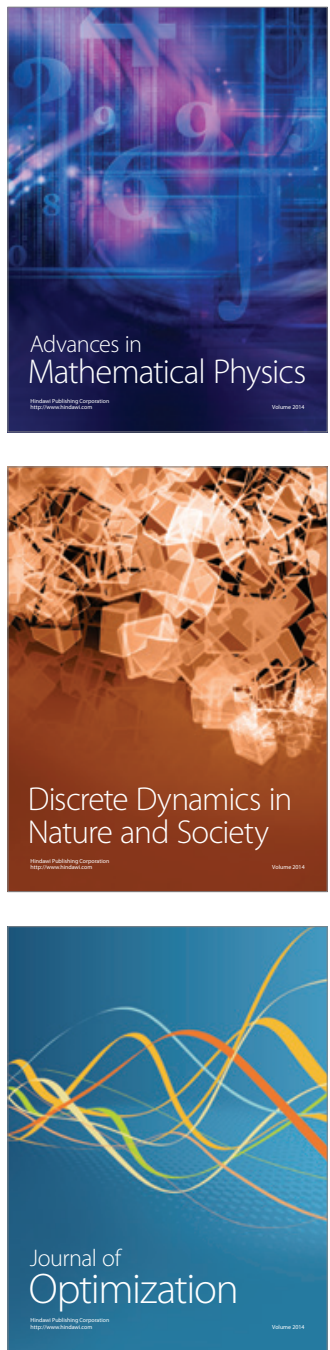